\title{
Early oral nutrition improves postoperative ileus through the TRPA1/CCK1-R-mediated mast cell-nerve axis
}

\author{
Da-Li Sun ${ }^{1,2}$, Yu-Xing $\mathbf{Q i}^{1,2 \#}$, Ting Yang ${ }^{1,2 \#}$, Yue-Ying Lin ${ }^{1,2}$, Shu-Min $\mathrm{Li}^{1,2}$, Yi-Jun Li ${ }^{1,2}$, Qing-Wen Xu ${ }^{1,2}$, \\ Yan-Bo Sun ${ }^{1,2}$, Wei-Ming Li ${ }^{1,2}$, Xiong-Zhi Chen ${ }^{1,2}$, Peng-Yuan $\mathrm{Xu}^{1,2}$ \\ ${ }^{1}$ Department of Gastrointestinal Surgery, Second Affiliated Hospital of Kunming Medical University, Kunming 650101, China; ${ }^{2}$ Yunnan Research \\ Center for Surgical Clinical Nutrition, Kunming 650101, China \\ Contributions: (I) Conception and design: DL Sun, PY Xu, T Yang, Q.P.L and YX Qi; (II) Administrative support: None; (III) Provision of study \\ materials or patients: None; (IV) Collection and assembly of data: DL Sun, YX Qi, T Yang, YY Lin, WM Li, QW Xu, YJ Li, SM Li, XZ Chen; (V) \\ Data analysis and interpretation: XZ Chen, DL Sun, PY Xu; (VI) Manuscript writing: All authors; (VII) Final approval of manuscript: All authors. \\ \#These authors contributed equally to this work. \\ Correspondence to: Xiong-Zhi Chen. Department of Gastrointestinal Surgery, Second Affiliated Hospital of Kunming Medical University, Kunming \\ 650101, China. Email: 13187878487@163.com; Wei-Ming Li. Department of Gastrointestinal Surgery, Second Affiliated Hospital of Kunming \\ Medical University, Kunming 650101, China. Email: liweiming49@163.com; Peng-Yuan Xu. Department of Gastrointestinal Surgery, Second \\ Affiliated Hospital of Kunming Medical University, Kunming 650101, China. Email: destiny1978@163.com.
}

Background: The mechanism of early oral nutrition that regulates the mast cell-nerve axis to improve postoperative ileus (POI) remains unclear. This study aims to investigate whether early oral nutrition can improve POI through Transient receptor potential ankyrin-1 (TRPA1)/cholecystokinin 1 receptor (CCK1-R) in the mast cell-nerve axis.

Methods: Experiment 1: Male Sprague-Dawley (SD) rats were randomly divided into the TRPA1 inhibitor + oral nutrition group (TI + ON + POI), oral nutrition group (ON + POI), POI group (POI) and sham surgery group (Sham). Nine rats in each group were treated. Experiment 2: Primary cultures of mast cells and dorsal root ganglion cells were created, and a non-contact co-culture system was established. The cells were divided into the dorsal root ganglion (DRG) group, mast cell group, DRG + mast cell group, TRPA1 inhibitor or enhancer group, mast cell stabilizer or enhancer group, CCK1-R inhibitor or enhancer group. The results of expression of TRPA1, CCK1-R and histamine in colon tissue, portal vein blood, supernatant or dorsal root ganglia, intestinal transport test and mast cell morphology were analysed.

Results: In experiment 1, Early oral nutrition could alleviate the degranulation and activation of mast cells and alleviate the inflammatory reaction of intestinal wall muscles $(\mathrm{P}<0.05)$. Early oral nutrition improved POI by stabilizing mast cells with TRPA1. TRPA1 inhibitor decreased CCK1-R concentrations in portal vein blood and CCK1-R expression in colonic smooth muscle $(\mathrm{P}<0.05)$. In experiment 2 , the change in mast cell function regulated the secretion of CCK1-R by neurons, CCK1-R negatively regulated the degranulation and activation of mast cells $(\mathrm{P}<0.05)$, and mast cells positively regulated the expression of TRPA1 protein in DRG $(\mathrm{P}<0.05)$.

Conclusions: Early enteral nutrition can improve POI through the TRPA1/CCK1-R-mediated mast cellnerve axis. TRPA1 positively regulates CCK1-R to stabilize mast cells, but TRPA1 is not the target of the downstream CCK1-R pathway.

Keywords: Early oral nutrition; postoperative ileus (POI); mast cell-nerve axis; transient receptor potential ankyrin-1 (TRPA1); cholecystokinin 1 receptor (CCK1-R)

Submitted Sep 23, 2019. Accepted for publication Jan 03, 2020.

doi: $10.21037 /$ atm.2020.01.95

View this article at: http://dx.doi.org/10.21037/atm.2020.01.95

(c) Annals of Translational Medicine. All rights reserved. 


\section{Introduction}

Postoperative ileus (POI) is a common complication after major abdominal surgery. POI is a short-term gastrointestinal peristalsis dysfunction after surgery. It manifests as short-term interruption of intestinal function, disappearance of bowel sounds, gastrointestinal effusion, abdominal pain and distention, nausea, vomiting and defecation delay, which can lead to increased complications, prolonged hospitalization and increased hospitalization cost (1).

The mast cell-nerve axis plays an important role in the pathogenesis of POI. Manipulating the intestinal tract during operation can lead to substantial mast cell activation and degranulation, release inflammatory mediators, and produce acute neurogenic responses (2). Anatomically, mast cells in intestinal mucosa are closely associated with vagal nerve endings (3). Electrical stimulation of the vagus nerve can reduce histamine release from mast cells; histamine is an important mediator triggering the inflammatory cascade reaction $(3,4)$. Mast cell degranulation mediates the infiltration of the leucocyte mucosal muscle layer after intestinal tract manipulation. Mast cell stabilizers can reduce the infiltration of the leucocyte mucosal muscle layer, alleviate intestinal inflammatory reactions and improve gastrointestinal motility $(5,6)$.

A physiologic approach to activate the cholinergic antiinflammatory vagal pathway is the administration of early enteral nutrition. However, how early enteral nutrition regulates the mast cell-nerve axis to improve POI is unclear.

Transient receptor potential ankyrin-1 (TRPA1) is an important cationic channel protein that exists in the cell membrane. TRPA1 is mainly expressed in the sensory nerve and mediates cold pain, mechanical and chemical damage stimuli $(7,8)$. The Cholecystokinin-1 receptor (CCK1-R) is an important receptor for cholecystokinin, which is mainly involved in the control of gastrointestinal smooth muscle movement and visceral sensation. In an animal study, highfat enteral nutrition was administered to a POI rat model, and the concentration of mast cell protease-II (MCP-II) was measured in plasma. As a result, high-fat enteral nutrition inhibited mast cell degranulation to a certain extent, while CCK receptor antagonist blocked this process (9). In other animal studies of a lymphatic fistula rat model, 20\% fat emulsion was administered to the duodenum, and the researchers detected histamine, MCP-II and prostaglandin D2 in lymph nodes. Compared with the control group given saline, the fat emulsion group exhibited activation of mast cells in the intestinal submucosa during the absorption of fat emulsion, and mast cell-related mediators entered the blood circulation (10). In another study, the researchers established an intestinal submucosal mast cell degranulation C57/Bl6 rat model by administering Salmonella lipopolysaccharide. Compared with low-fat enteral nutrition and fasting, highfat enteral nutrition significantly reduced circulating mast cell protease-I (MCP-I), but CCK1-R blocker, nAChR blocker and removal of the intestinal vagus nerve can eliminate the effect of high-fat enteral nutrition (11). Some studies have confirmed that TRPA1 participates in the recovery of POI $(12,13)$. Unsaturated fatty acids in enteral nutrition can stimulate the release of cholecystokinin (CCK) secreting cells through TRPA1, thus regulating intestinal motility (14). Although the models used and the results obtained in these studies are different, to some extent, it can be inferred that enteral nutrition and its nutrients can regulate the mast cell-nerve axis. Additionally, CCK1-R and TRPA1 could possibly be involved in this process.

Therefore, the purpose of this study was to explore the role of TRPA1/CCK1-R in early enteral nutrition to improve POI through the mast cell-nerve axis by establishing a rat model of POI and a non-contact coculture system of mast cells and nerve cells. Additionally, we aimed to confirm the relationship between TRPA1 and CCK1-R.

\section{Methods}

\section{Experiment 1}

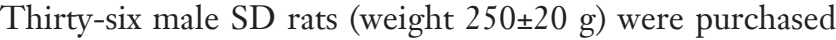
from Kunming Medical University. All rats were kept in stainless steel cages with controllable indoor temperature and humidity. During the adaptation period of 7 days before the operation, rats were free to consume water and rodent food. All experiments were conducted in accordance with the care and use of laboratory animals published by the Ministry of Science and Technology of China and approved by the Ethics Committee of Kunming Medical University.

\section{Modelling}

Twelve hours before the operation, rats were fasted, but free water intake was permitted. Before the experiment, all the instruments were sterilized and dried in the oven. Medical disinfection alcohol (75\%) was sprayed on the table, and the experiment was started after the alcohol volatilized to the table drying. Rats were placed into the anaesthesia machine, 
the oxygen flow rate was adjusted to $50 \mathrm{kPa}$, and $2 \%$ isoflurane was inhaled at a $0.3 \mathrm{~mL} /$ minute flow rate through an anaesthesia mask. After anaesthesia was administered to the rats, the abdominal coat was shaved with a small animal shaver and disinfected with iodophor. Rats were fixed on the operating table in the supine position and connected to a respirator mask to maintain anaesthesia.

\section{Rat model of POI}

A 2-cm longitudinal incision was made along the midline of the abdomen, one cotton swab was held in each hand, and the colon was gently pulled out with a cotton swab and carefully placed on damp gauze. A cotton swab was used to turn the colon carefully back and forth for 5 minutes, and the manipulation was gently performed to avoid breaking the capillaries around the intestinal tract. Then, the colon was carefully returned to the abdominal cavity, and the abdomen was closed after disinfection with iodophor. Next, the respirator was closed, the respirator mask was removed, and the rat was placed in a cage after awakening and given adequate food and water.

\section{Sham-operated rat model}

Except for the lack of manipulation of the intestinal tract, the other processes were the same as those for the POI model described above.

\section{Animal grouping and intervention}

Thirty-six male SD rats were randomly divided into the TRPA1 inhibitor + oral nutrition group $(\mathrm{TI}+\mathrm{ON}+\mathrm{POI})$, oral nutrition group (ON + POI), POI group (POI) and sham surgery group (sham) with 9 rats in each group. TRPA1 inhibitor + early oral nutrition group (TI + ON + POI): After the establishment of the POI model, rats were given an intraperitoneal injection of TRPA1 inhibitor HC030031 (200 mg/kg body weight). At 5, 45 and 90 minutes after the operation, $0.75 \mathrm{ml}$ of enteral nutrition solution (Peptisorb, Nutricia Ltd, Wuxi, China) was given forcibly. In the early oral nutrition group (ON + POI), $0.75 \mathrm{~mL}$ enteral nutrition solution (Peptisorb) was given forcibly at 5, 45 and 90 minutes after operation. POI group (POI): a POI model was established, and samples were extracted for detection. Sham operation group (sham): Sham operation was performed, and samples were extracted for detection.

\section{Sampling}

At $6 \mathrm{~h}$ after the operation, the abdominal cavity was opened in 6 rats to obtain portal vein blood samples under anaesthesia. After death caused by excessive anaesthesia, fresh colonic tissue (approximately $1 \mathrm{~cm}$ ) was washed with sterile and RNase-free water. Colonic mucosa and colonic smooth muscle tissue were separated and frozen in cryopreservation tubes at $-80^{\circ} \mathrm{C}$. The spinal cord (the lumbar dorsal root ganglion (DRG) was separated) was frozen and stored at $-80^{\circ} \mathrm{C}$.

\section{Real-time quantitative PCR (RT-qPCR)}

The expression of CCK1-R, histamine receptors (HRH1 and HRH4) in colonic smooth muscle tissue, TRPA1 in colon, and TRPA1 in lumbar DRG were detected. Primer design and synthesis: PubMed was used to search the target gene RNA sequence of the corresponding species, and the cording sequence was used to design primers. RT-qPCR primers were designed by beacon designer (version 7.90 Premier Biosoft International, Palo Alto, CA, USA). The sequence of primers is shown in Table S1. According to the manufacturer's instructions, total RNA was extracted from colonic tissue, colonic smooth muscle and lumbar DRG by using TRIZOL lysate (Invitrogen). Then, the concentration of $3 \mu \mathrm{L}$ total RNA was measured in an ND-1000 spectrophotometer. The volume of total RNA required for reverse transcription was calculated and synthesized according to a Revert Aid ${ }^{\mathrm{TM}}$ First Strand cDNA Synthesis Kit. RT-qPCR was then performed in a LightCycler 96 Real-Time PCR System (Roche Diagnostics, Germany). Data were analysed by relative quantification of gene expression (LightCycler ${ }^{\circledR} 96$ software, Roche Diagnostics, Germany) and standardized to the level of $\beta$-actin transcription.

\section{Western blot analysis}

TRPA1 in colon mucosa, CCK1-R, TRPA1 in colon tissue and TRPA1 in lumbar DRG were detected. Total protein was extracted using standard protocols, and lysates were resolved via electrophoresis on sodium dodecyl sulphate polyacrylamide gel electrophoresis (SDS-PAGE) gels. Separated proteins were transferred to a polyvinylidene fluoride (PVDF) membrane (Bio-Rad Laboratories, Inc., CA, USA) for immunoblotting using a wet-transfer system. Following transfer, membranes were incubated overnight at $4{ }^{\circ} \mathrm{C}$ with primary antibodies (diluted $1: 1,000$ ), and bound antibody was detected using HRP-conjugated secondary antibodies $(1: 2,000$ or $1: 4,000$; Bio-Rad Laboratories). HRP-bound antibodies were then detected on film via enhanced chemiluminescence (GE Healthcare, IL, USA). 
Enzyme-linked immunosorbent assay (ELISA)

The contents of MCP-II and CCK1-R in portal vein blood were detected. The tissue was extracted according to the standard scheme, and the contents of MCP-II and CCK1-R in the samples were calculated by ELISA Calc software according to the manufacturer's instructions.

\section{Intestinal transport experiment}

Six hours after the operation, three rats in each group were fed fluorescein isothiocyanate (FITC)-Dextran (dissolved in normal saline) labelled with FITC at $0.6 \mathrm{~g} / \mathrm{kg}$ body weight. After 90 minutes, fresh small intestine and colon were taken. Ten segments of the intestinal tract and its contents were taken from the small intestine, each of which was approximately $1 \mathrm{~cm}$ in length. Three segments of colon and its contents were taken, each of which was approximately $1 \mathrm{~cm}$ in length. Intestinal content of each segment was added with $500 \mu \mathrm{L}$ PBS solution and centrifuged at $5,000 \mathrm{rpm}$ for 5 minutes, and $200 \mu \mathrm{L}$ supernatant was taken. The supernatant samples were added to a 96-well enzyme plate. FITC fluorescence was measured at $520 \mathrm{~nm}$ excitation wavelength and $485 \mathrm{~nm}$ emission wavelength. The distribution of FITC-labelled glucan in the gastrointestinal tract was calculated by calculating the geometric centre. The formula was geometric centre $(\mathrm{GC})=\Sigma$ (percentage of fluorescein per segment $\times$ number of segments)/100 (9).

\section{Morphology of mast cells observed by transmission electron microscopy}

Fresh colonic tissues were extracted and fixed at $4{ }^{\circ} \mathrm{C}$ with 2.5\% glutaraldehyde, washed with PBS buffer, embedded and cured by Epon 812 epoxy resin, sliced by an UltracutE ultrathin slicing machine and stained by uranium acetate and lead nitrate. Then, the morphology of mast cells was observed under transmission electron microscopy.

\section{Experiment 2}

\section{Mouse primary mast cell culture}

Bone marrow-derived mast cells (BMMCs) were grown by culturing bone marrow cells from male $\mathrm{SD}$ rats as previously reported $(15,16)$. Briefly, bone marrow cells were aspirated and cultured in DMEM/F12 supplemented with interleukin-3 (IL-3) (30 ng/mL), colony stimulating factor (CSF) $(20 \mathrm{ng} / \mathrm{mL}), 10 \%$ heat-inactivated fetal bovine serum (FBS) (15 mL), 1\% penicillin-streptomycin, $0.07 \%$ beta-mercaptoethanol, and $1 \% \mathrm{~L}$-glutamine for 4 weeks at $37{ }^{\circ} \mathrm{C}$ in a $5 \% \mathrm{CO}_{2}$ incubator. During this culture period, non-adherent cells were removed once every 2 or 3 days with the addition of fresh culture medium. After 4 weeks of culture, mast cells were identified by $0.1 \%$ toluidine blue staining (17) (see attached Figure 1).

\section{Primary culture of rat DRG neurons}

Dorsal root ganglia were removed from the lumbar spinal cord of male SD rats $(150 \pm 200 \mathrm{~g})$, and cultures were prepared based on the method described by Pongrac and Rylett (18). In brief, rats were anaesthetized with inhaling isoflurane $(2 \%, 0.3 \mathrm{~mL} / \mathrm{minute}$ by an anaesthesia mask) and dissected following procedures approved by the Animal Research Ethics Committee of Kunming Medical University. DRG were dissected into DMEM/F12 medium (DMEM/F12 containing $10 \mathrm{~mL} \mathrm{10 \%} \mathrm{heat-inactivated}$ FBS, $1 \mathrm{~mL}$ double antibody, $1 \mathrm{~mL} \mathrm{1 \%} \mathrm{L-glutamine)} \mathrm{and}$ incubated for 24 hours $\left(37^{\circ} \mathrm{C}, 5 \% \mathrm{CO}_{2}\right)$. Neurobasal A medium (including $12 \mathrm{~mL} \mathrm{~B} 27,1 \mathrm{~mL}$ double antibody, $1 \mathrm{~mL} \mathrm{1 \%} \mathrm{L-glutamine)} \mathrm{from} \mathrm{the} \mathrm{second} \mathrm{day} \mathrm{was} \mathrm{replaced,}$ and DRG neurons were maintained in culture for 6 days in an atmosphere of $5 \% \mathrm{CO}_{2}$ at $37^{\circ} \mathrm{C}$. After 7 days of culture, DRG neurons were detected by immunofluorescence assay. (see attached Figure 2).

\section{Mast cell and DRG neuron non-contact co-culture and intervention}

DRG group (Control-N): the DRG neurons were cultured alone.

Mast cell group (Control-M): Mast cells were cultured alone.

DRG and mast cell group (Control-N+M): Mast cells were inoculated into an upper Transwell chamber with $10^{5}$ cells in each pore and DRG neurons in the lower chamber. During co-culture, 1640 basic medium was used for mast cells in the superior chamber, and Neurobasal A basic medium was used for DRG neurons in the inferior chamber.

TRPA1 inhibitor group (-TRPA1): DRG neurons were pre-treated with HC-030031 at a final concentration of $50 \mu \mathrm{mol} / \mathrm{L}$ for 16.5 minutes $(19,20)$ and non-contact cocultured with mast cells.

Mast cell stabilizer group (-Mast cell): mast cells were pre-treated with sodium glycyrrhizinate at a final concentration of $100 \mu \mathrm{M} / \mathrm{L}$ for 30 minutes $(21,22)$ and noncontact co-cultured with DRG neurons.

CCK1-R inhibitor group (-CCK1-R): mast cells were non-contact co-cultured with DRG neurons after treatment with rebamipide at a final concentration of $37.7 \mathrm{nmol} / \mathrm{L}$ for an hour (23). 

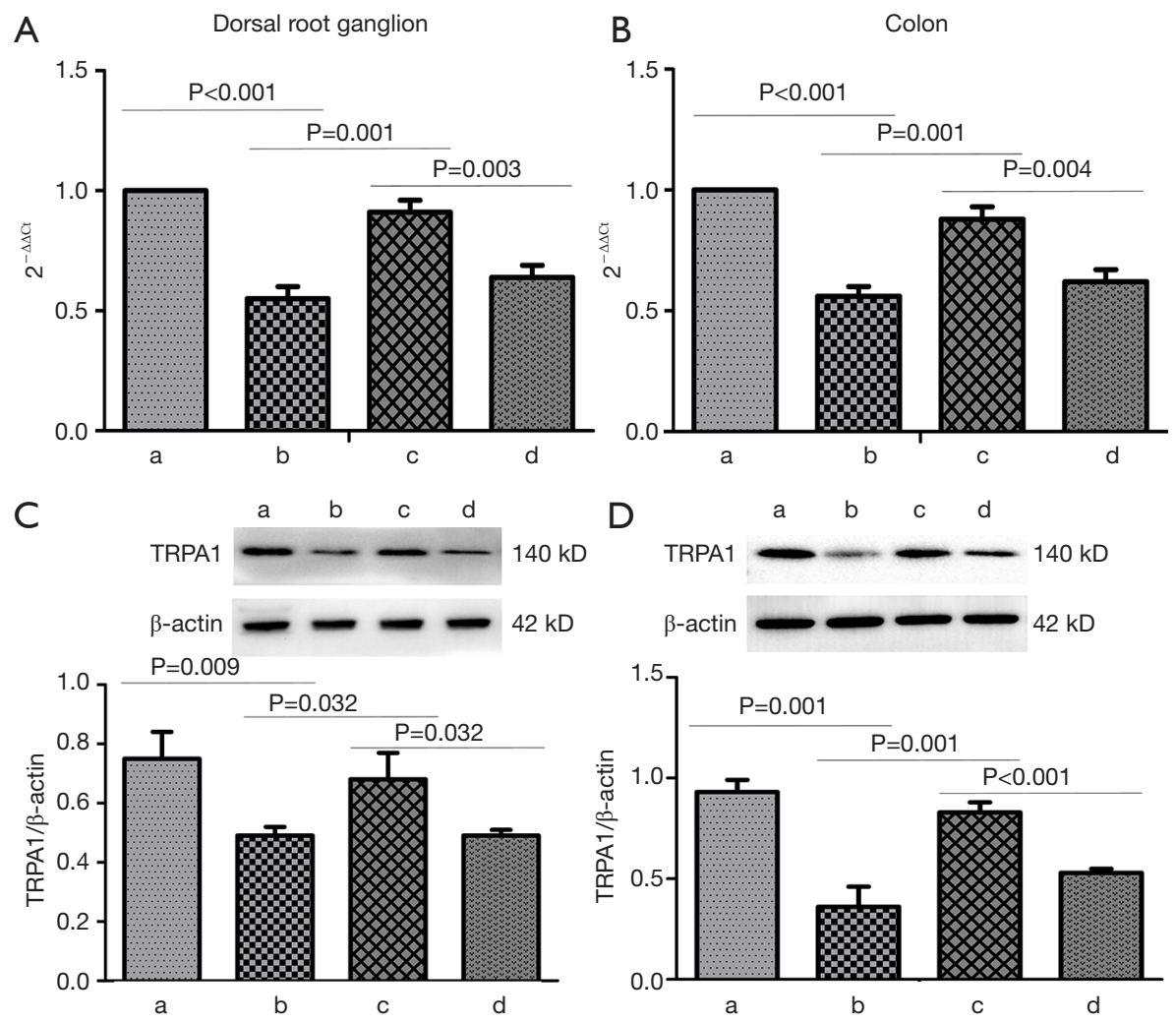

Figure 1 Expression of TRPA1 mRNA and protein in dorsal root ganglion and colon. (A) Expression of TRPA1 mRNA in dorsal root ganglion. (B) Expression of TRPA1 mRNA in colon. (C) Expression of TRPA1 protein in dorsal root ganglion. (D) Expression of TRPA1 protein in colon. (a), (b), (c) and (d) represent sham surgery group, POI group, oral nutrition group and TRPA1 inhibitor + oral nutrition group respectively.
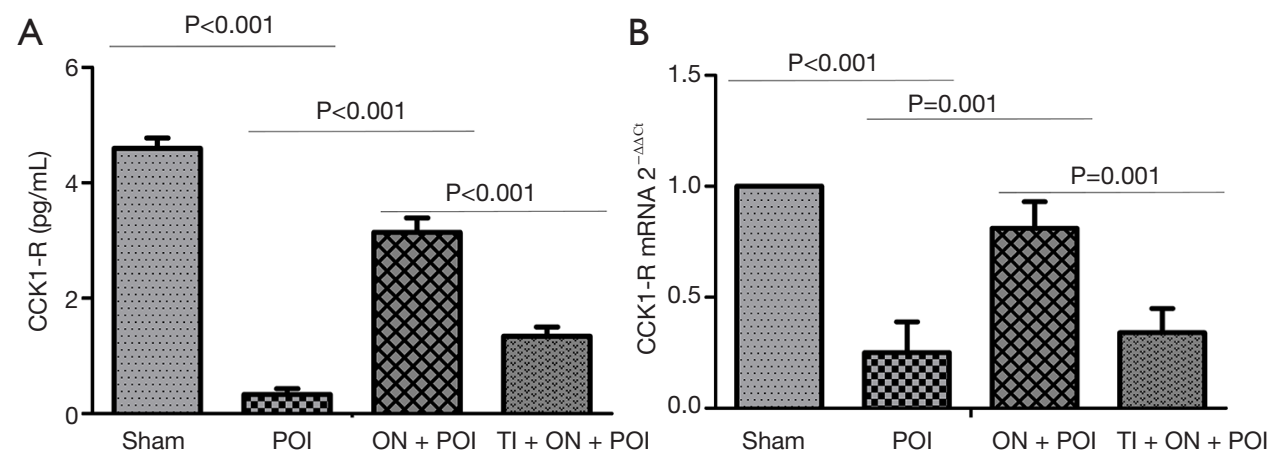

Figure 2 CCK1-R concentration in portal vein blood (A) and CCK1-R expression in the smooth muscle layer of the colon (B). Sham, POI, $\mathrm{ON}+\mathrm{POI}$ and TI + ON + POI represent sham surgery group, POI group, oral nutrition group and TRPA1 inhibitor + oral nutrition group respectively.

TRPA1 enhancer group (+TRPA1): DRG neurons were non-contact co-cultured with mast cells after treatment with allyl thiocyanate at $10 \mu \mathrm{mol} / \mathrm{L}$ for 15 minutes $(24,25)$.

Mast cell enhancer group (+Mast cell): mast cells were non-contact co-cultured with DRG neurons after treatment with lipopolysaccharide (LPS) at a final concentration of $100 \mathrm{ng} / \mathrm{mL}$ for an hour $(26,27)$.

CCK1-R enhancer group (+CCK1-R): mast cells 
were pre-treated with A-71623 at a final concentration of $3.7 \mathrm{nmol} / \mathrm{L}$ for an hour (28) and non-contact co-cultured with DRG cells.

\section{Sampling}

The supernatant was collected $48 \mathrm{~h}$ after culture in the above 9 groups, CCK1-R and histamine were detected by ELISA, and DRG neurons were collected from DRG (in Control-M group, mast cells were collected) to detect TRPA1 protein expression by Western blot analysis. The expression of TRPA1 in DRG neurons or mast cells was detected by RT-qPCR. The detection method was the same as that in experiment 1 .

\section{Statistical analysis}

Statistical analysis was processed by SPSS17.0 software (SPSS Inc., Chicago, USA). The measurements are expressed as the mean \pm standard deviation (SD), and $t$-test was used to compare the two groups. The experimental data obtained by RT-QPCR were processed by $2^{-\triangle \Delta \mathrm{Ct}}$ analysis on the premise that the amplification efficiency of the target gene was similar to that of the internal reference gene. The Ct value refers to the number of cycles that the fluorescent signal in each reactor tube experiences when it reaches the set threshold. The threshold is negatively correlated with the expression level of the sample. The increase in the Ct value indicates that the expression level of the sample decreases. The average $\mathrm{Ct}$ value, $\Delta \mathrm{Ct}$ value and $\Delta \Delta \mathrm{Ct}$ value of each sample were calculated. $\Delta \mathrm{Ct}$ value $=$ target gene Ct value - internal reference gene CT value, $\Delta \Delta \mathrm{Ct}$ value $=$ experimental group $\Delta \mathrm{Ct}$ mean value - control group $\Delta \mathrm{Ct}$ mean value, calculated $2^{-\Delta \Delta \mathrm{Ct}}$ value used to represent the relative multiple of the experimental group compared with that of the control group.

\section{Results}

\section{TRPA1 and CCK1-R are involved in the occurrence of POI}

TRPA1 is mainly expressed in sensory nerve endings. In experiment 1 , POI models were established as previously described (9). Compared with the sham group, the POI group exhibited significantly decreased expression of TRPA1 in the DRG and colon tissue $(\mathrm{P}<0.05)$ (Figure 1). CCK1-R is also expressed in neurons and can be released outside the cells. Compared with the sham group, the POI group showed a significant decrease in the CCK1-R concentration in portal vein blood and CCK1-R expression in the smooth muscle layer of the colon $(\mathrm{P}<0.05)$ (Figure 2). These data suggested that TRPA1 and CCK1-R were involved in the occurrence of POI.

\section{Early oral nutrition improved mast cell degranulation and postoperative ileus}

Intestinal manipulation initiates an inflammatory response in the intestinal muscularis, which results from activation and degranulation of mast cells $(5,9)$. MCP-II is a hydrolase expressed in mucosal mast cells and is often used as a marker of mast cell activation or degranulation. The concentration of MCP-II in the portal vein blood of the $\mathrm{ON}+\mathrm{POI}$ group was significantly lower than that in POI group $(\mathrm{P}<0.001)$ (Figure 3$)$. Mast cells contain a large amount of histamine, which is often released after activation of mast cells. Therefore, the detection of histamine content can indirectly indicate the degree of mast cell activation and degranulation, and histamine also acts as an inflammatory mediator in intestinal smooth muscle. Because histamine is a self-activating substance, which is formed by the decarboxylation of histidine in vivo, there is no gene encoding histamine. Histamine can indirectly react with the content of histamine by detecting its receptor. At 6 hours after operation, HRH1 and HRH4 mRNA in the $\mathrm{ON}+\mathrm{POI}$ group were significantly lower than those in the POI group $(\mathrm{P}<0.05)$ (Figure 3). Indirectly, early oral nutrition can reduce histamine release caused by intestinal manipulation and, to some extent, reduce the level of inflammatory response. Electron microscopy of mast cells also showed that the degranulation degree of mast cells significantly increased in the POI group. Additionally, the degranulation degree of mast cells in the $\mathrm{ON}+\mathrm{POI}$ group was significantly lower than that in the POI group (Figure 4). These data suggest that enteral oral nutrition can reduce degranulation and activation of mast cells and alleviate inflammation in intestinal wall muscle layers.

\section{Early oral nutrition stabilized mast cells through TRPA1 to improve POI}

It has been proven that high-fat enteral nutrition can improve POI by activating CCK1-R (9). Therefore, this part of the experiment focused only on whether enteral nutrition may improve POI by activating TRPA1. In experiment 1 , after establishing the POI model, early oral nutrition could increase the geometric centre $(\mathrm{P}=0.021)$ 

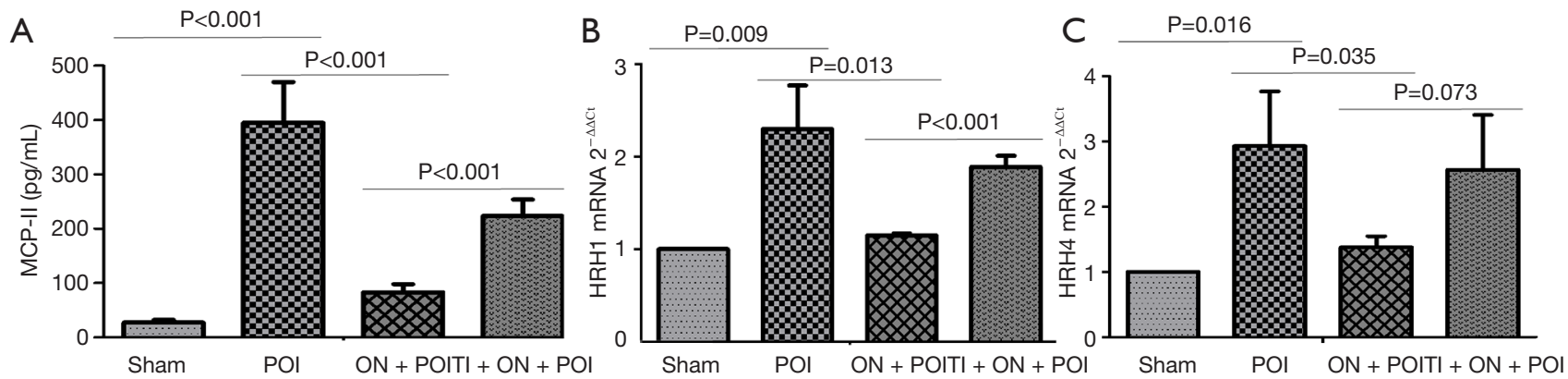

Figure 3 The concentration of MCP-II in the portal vein blood (A) and expression of HRH1 and HRH4 mRNA in colonic smooth muscle tissue (B and C). Sham, POI, ON + POI and TI + ON + POI represent sham surgery group, POI group, oral nutrition group and TRPA1 inhibitor + oral nutrition group respectively.

(Figure 5). Following the addition of TRPA1 inhibitor, the effect of enteral nutrition on increasing the geometric centre after operation was weakened $(\mathrm{P}=0.045)$ (Figure 5). This result suggests that enteral nutrition can improve POI through TRPA1.

To further clarify whether enteral nutrition stabilizes mast cells through TRPA1, we administered TRPA1 inhibitors after early oral nutrition intervention in experiment 1. Degranulation and activation of mast cells were observed. TRPA1 inhibitors reversed the effects of early oral nutrition on MCP-II concentration in portal vein blood, the expression of HRH1 and HRH4 mRNA in colonic smooth muscle $(\mathrm{P}<0.05)$ (Figure 3) and morphological changes in mast cell degranulation in the intestinal tissue (Figure 4). These data suggest that enteral nutrition improves POI by stabilizing mast cells with TRPA1.

\section{TRPA1 mediated information transmission between mast cells and nerve cells through CCK1-R}

In experiment 1 , the concentration of CCK1-R in portal vein blood and the expression of CCK1-R in intestinal smooth muscle significantly decreased following administration of TRPA1 inhibitor in the TI + ON + POI group compared with that in the $\mathrm{ON}+\mathrm{POI}$ group $(\mathrm{P}<0.05)$ (Figure 2). This finding indicates a regulatory relationship between TRPA1 and CCK1-R.

To further verify the information transmission between mast cells and nerve cells by TRPA1 and CCK1-R, we designed experiment 2 . After primary culture of mast cells and nerve cells, the cells were identified (Figure S1 and Figure S2), and then the two cell types were co-cultured in a non-contact manner. The concentration of CCK1-R in the supernatant and the expression of TRPA1 in neurons were detected. The concentration of histamine in the supernatant indirectly reacted with degranulation of mast cells. TRPA1 can positively regulate the concentration of CCK1-R in the supernatant by using TRPA1 enhancers and inhibitors (Figure 6). By incubating mast cells with mast cell enhancers and stabilizers, we determined that activation of mast cells could also regulate the concentration of CCK1-R in the supernatant, indicating that mast cells could regulate the neuronal secretion of CCK1-R (Figure 6).

Animal model studies have confirmed that CCK1-R antagonists can promote mast cell degranulation and activation (9). To further elucidate the regulatory effect of CCK1-R on the degranulation and activation of mast cells, in experiment 2, we incubated mast cells with CCK1-R enhancers and inhibitors. As a result, CCK1-R enhancers significantly reduced the histamine level in the supernatant of the culture medium, and CCK1-R inhibitors significantly reversed this effect $(\mathrm{P}<0.05)$ (Figure 6). Thus, CCK1-R can regulate the degranulation and activation of mast cells.

In experiment 2, to determine whether the effect of mast cell function on neurons was accomplished by TRPA1 and CCK1-R, we altered mast cell function by administering mast cell stabilizers and enhancers. Additionally, the expression of TRPA1 protein in the DRG and the concentration of CCK1-R in the supernatant were detected (Figure 6). The mast cell stabilizer increased TRPA1 expression in the DRG $(\mathrm{P}<0.05)$, while the mast cell enhancer could decrease TRPA1 expression in the DRG $(\mathrm{P}<0.05)$, indicating that the change in mast cell function could regulate TRPA1 expression in neurons (Figure 6). 

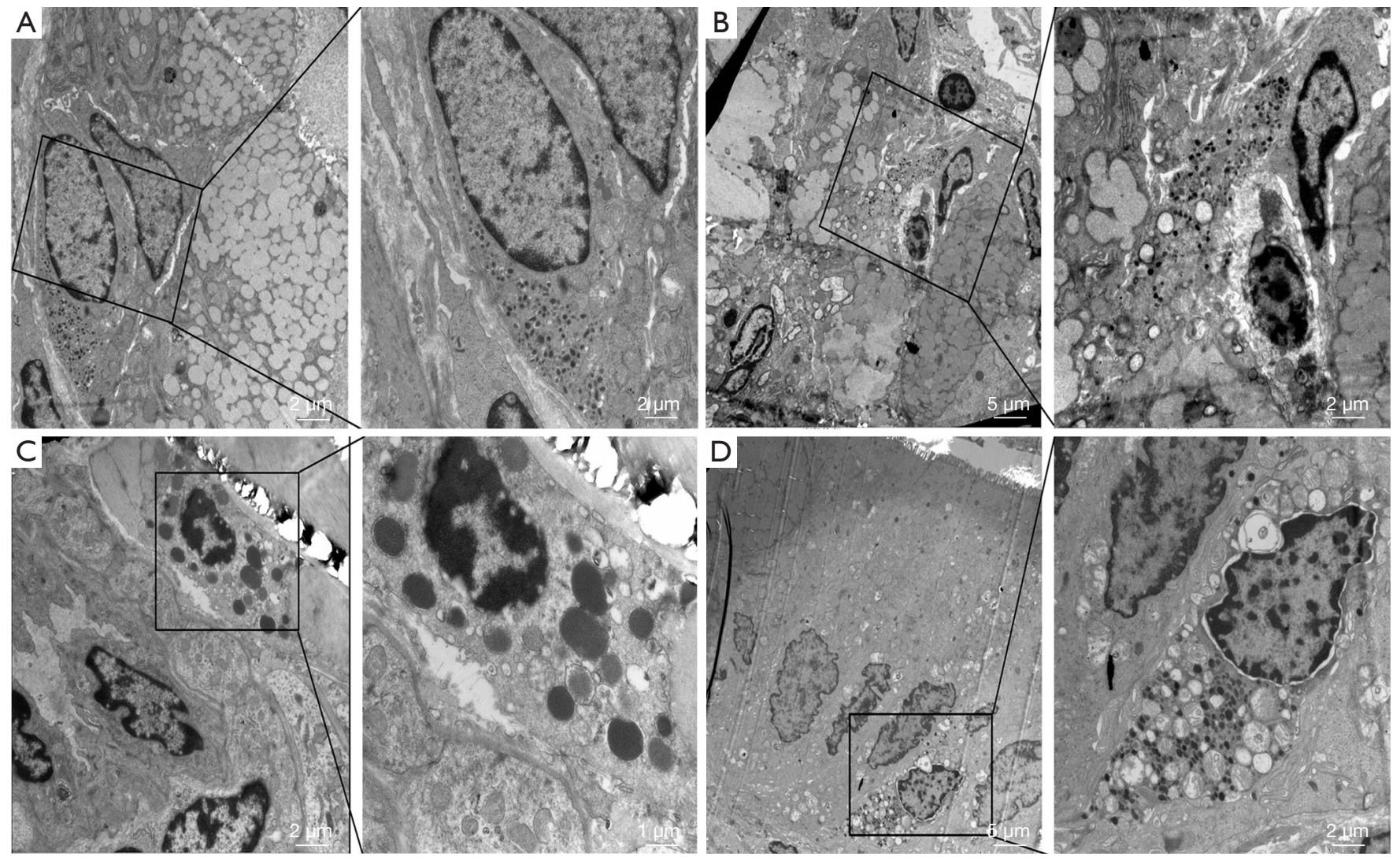

Figure 4 Morphology of mast cells of groups observed after staining with uranyl acetate and lead citrate by transmission electron microscopy. A, B, C and D represent sham surgery group, POI group, oral nutrition group and TRPA1 inhibitor + oral nutrition group respectively.
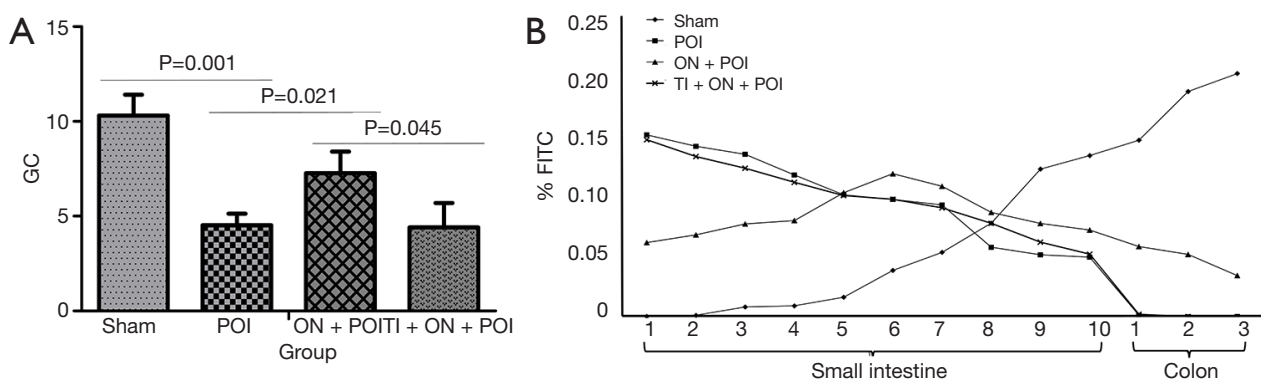

Figure 5 Geometric centre (GC) (A) and distribution of FITC fluorescence (B) in the gastrointestinal tract. Sham, POI, ON + POI and TI $+\mathrm{ON}+\mathrm{POI}$ represent sham surgery group, POI group, oral nutrition group and TRPA1 inhibitor + oral nutrition group respectively.

The concentration of CCK1-R in the supernatant was measured, revealing that the concentration of CCK1-R in the supernatant was significantly changed after mast cell stabilizers and enhancers were administered $(\mathrm{P}<0.05)$. These findings indicate that the change in mast cell function could regulate the secretion of CCK1-R by neurons (Figure 6). Following incubation of mast cells with CCK1-R inhibitors and enhancers, no significant difference was observed in the expression of TRPA1 protein in the DRG $(\mathrm{P}>0.05)$, suggesting that TRPA1 may not be the downstream target of the CCK1-R pathway (Figure 6). 


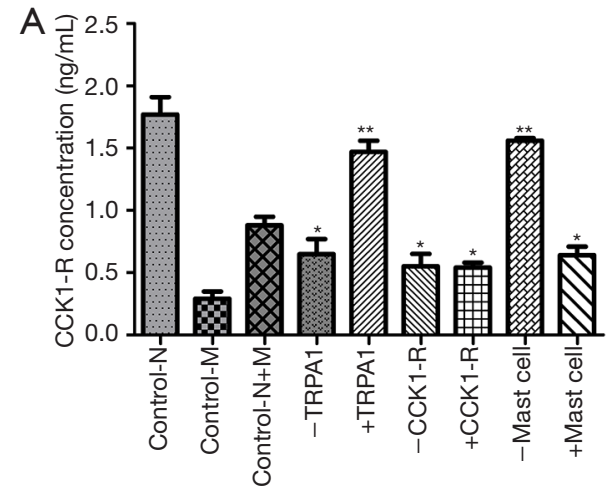

C

Groups

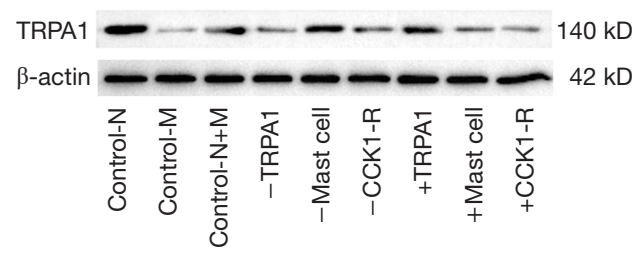

Groups

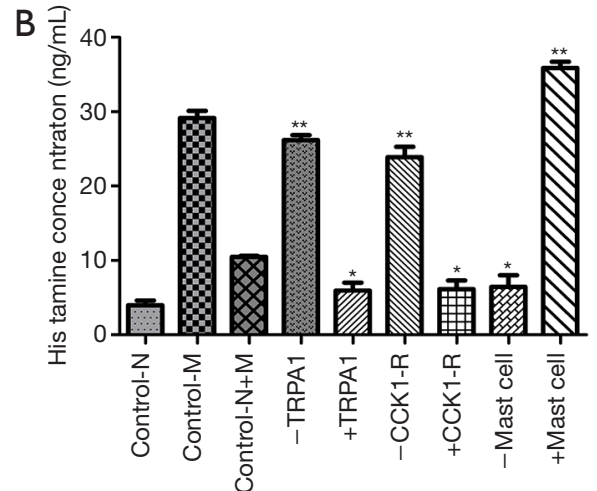

Groups

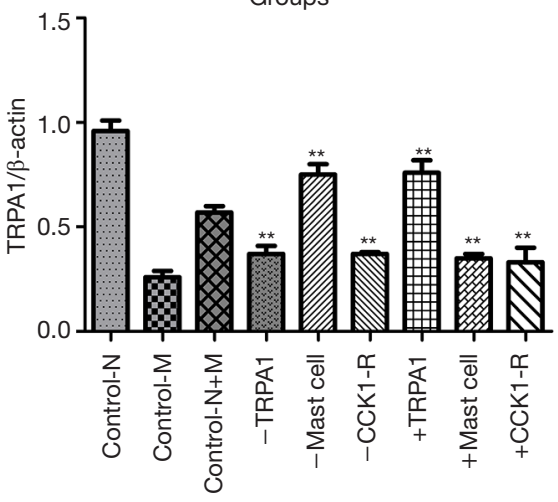

Groups

Compared with Control-N+M group: ${ }^{*}, \mathrm{P}<0.05,{ }^{* *}, \mathrm{P}<0.05$

Figure 6 CCK1-R (A) and histamine (B) concentration in supernatant and expression of TRPA1 protein (C) and mRNA (D) in neurons. Control-N, Control-M, Control-N+M, -TRPA1, -Mast cell, -CCK1-R, +TRPA1, +Mast cell and +CCK1-R represent DRG group, Mast cell group, DRG and mast cell group, TRPA1 inhibitor group, Mast cell stabilizer group, CCK1-R inhibitor group, TRPA1 enhancer group, Mast cell enhancer group and CCK1-R enhancer group respectively.

\section{Discussion}

In this study, we established a rat POI model to evaluate whether early oral nutrition can improve POI through the TRPA1/CCK1-R-mediated mast cell-nerve axis. Then, we further validated the role of TRPA1/CCK1-R in mast cell and nerve cell information transmission through a non-contact co-culture system of mast cell neurons. As anticipated, early oral nutrition improved postoperative intestinal paralysis by TRPA1/CCK1-R mediating the mast cell-nerve axis.

\section{Effects of early oral nutrition on mast cell activation and degranulation in POI}

It has been proven that activation and degranulation of mast cells are important links in the occurrence of POI $(2,9)$.
In this study, degranulation and activation of mast cells were found in POI, similar to findings in previous studies $(5,9)$. Mast cell infiltration occurs in the early stage of POI, and degranulation of mast cells is an important step of leukocyte infiltration because specific mast cell stabilizers can prevent intestinal leukocyte infiltration and delayed gastric emptying after intestinal manipulation (5). In clinical and animal studies, stabilizing mast cells and inhibiting macrophages improved intestinal peristalsis caused by inflammatory reactions in POI $(5,6,29)$. Moreover, intestinal inflammation induced by intestinal manipulation was significantly reduced in the mast cell gene knockout mouse model (5). Histamine is an important inflammatory mediator that initiates inflammation. In this study, the expression of histamine receptor mRNA in smooth muscle was detected to indirectly show the content of histamine. The expression of HRH1 and HRH4 mRNA in smooth 
muscle tissue of colon with POI was significantly increased, which indirectly indicated that POI resulted in increased degranulation and secretion of histamine from mast cells.

In this study, early oral nutrition reduced mast cell activation and degranulation in POI. The following possible reasons may explain the results. In a previous animal model study, the researchers compared high-fat enteral nutrition with conventional enteral nutrition. The fat in enteral nutrition was beneficial to decrease the concentration of MCP-II in the portal vein of rats with POI (9). This finding may partly explain the effect of early oral nutrition on the activation and degranulation of mast cells in POI. Another cause of concern is that early oral nutrition stimulates visceral nerves and regulates mast cell function. Intestinal mast cells are very close to the vagal nerve endings anatomically, and mast cell function can be affected by stimulating the vagus nerve $(30,31)$.

\section{TRPA1 was involved in the occurrence of POI, and early oral nutrition regulated the expression of TRPA1}

In this study, the expression of TRPA1 in colon tissue and DRG was significantly decreased in POI. Early oral nutrition could promote the expression of TRPA1. After adding TRPA1 inhibitors, the intestinal transport capacity was decreased, similar to findings in previous studies $(12,13)$. In a study of a male C57Bl/6 rat model of POI (12), the authors used TRPV1 agonist capsaicin, TRPA1 agonist allyl isothiocyanate (AITC), cinnamaldehyde (CA), TRPA1/ TRPV1 agonist daikenchuto Dajianzhong decoction (TU100) and 6-shogaol (6SG) for intervention and found that TRPA1 agonists (AITC, CA, TU-100 and 6SG) significantly improved slow gastrointestinal transport, while capsaicin (TRPV1 agonist) had no such effect. The authors considered that TRPA1 is necessary to induce and alleviate POI. In another study, TPRA1 agonists promoted intestinal transport in rats with POI (13).

How enteral nutrition enhances TRPA1 expression remains unclear. In an in vitro/in vivo study, polyunsaturated fatty acids in diet (including DHA, EPA, linolenic acid, etc.) can activate TRPA1 to stimulate primary neurons and endocrine cells, and this effect is absent in TRPA1 gene knockout mice. Continuous stimulation with polyunsaturated fatty acids can desensitize TRPA1. Therefore, researchers believe that TRPA1 is necessary for polyunsaturated fatty acids to stimulate primary neurons and endocrine cells (32).

\section{TRPA1 regulated mast cell activation and degranulation through CCK1-R}

Few studies have focused on TRPA1 regulating mast cell activation and degranulation, and we identified only one study in the PubMed database. Instead, researchers have focused on the effect of TRPA1 on airway hyperresponsiveness induced by activated mast cells (33). In this study, TRPA1 inhibitors were administered based on the implementation of early oral nutrition. Based on the results, TRPA1 inhibitors could inhibit the effect of early oral nutrition on stabilizing mast cells.

To further explore the mechanism of early oral nutrition regulating mast cell degranulation through TRPA1, we administered TRPA1 inhibitor and early oral nutrition simultaneously, and the concentration of CCK1-R in portal vein blood and the expression of CCK1-R mRNA in colonic smooth muscle were detected. After early oral nutrition, the CCK1-R concentration in portal vein blood and CCK1-R mRNA expression in colonic smooth muscle were increased. The expression of mRNA in colonic smooth muscle was significantly increased, and TRPA1 inhibitor significantly blocked this effect, suggesting that TRPA1 may regulate mast cell activation and degranulation through CCK1-R. In previous studies, a mucosal mast cell degranulation model was established in C57/B16 mice by administration of Salmonella enterica LPS. Compared with low-fat enteral nutrition (16\% energy supply from soybean lecithin) and fasting, high-fat enteral nutrition $(50.4 \%$ energy supply from soybean lecithin, $\omega-3$ and $\omega-6$ fatty acid content $<5 \%)$ significantly reduced the MCP-I concentration in circulation; however, CCK1-R blockers can eliminate the effect of high-fat enteral nutrition (11), which indicates that CCK1-R regulates mast cell degranulation. Therefore, TRPA1 mediates the effect of early oral nutrition on the degranulation of mast cells via CCK1-R in POI.

\section{TRPA1 and CCK1-R participated in information transmission between mast cells and neurons}

To further verify that TRPA1 and CCK1-R participate in information transmission between mast cells and neurons, we first designed a non-contact co-culture system for mast cells and neurons. In previous studies, researchers used contact co-culture to explore mast cell-neuron crosstalk (34-36). Researchers mainly focused on mast cell and neuron anatomical contact; however, our study determined 
that TRPA1 and CCK1-R are involved in the regulation of the mast cell-nerve axis. Because TRPA1 and CCK1-R are mainly expressed in neurons, mast cells were placed in the upper chamber and nerve cells in the lower chamber.

In this study, TRPA1 and CCK1-R negatively regulated mast cell degranulation, and mast cell activation or degranulation inhibits the expression of TRPA1 protein and the secretion of CCK1-R in neurons, indicating that TRPA1 and CCK1-R are involved in mast cell and nerve cell information transmission. In previous animal studies on CCK regulating mast cell degranulation, researchers used soybean trypsin inhibitors to induce endogenous CCK release in rats given CCK2-R antagonist (L-365260) intervention and detected MCP-II to evaluate mast cell degranulation. According to the results, endogenous CCK could regulate intestinal mucosal mast cell degranulation by stimulating CCK2-R. Researchers believe that intestinal mucosal mast cells are involved in CCK regulation of intestinal function (37). Although our study also suggested that the CCK signalling pathway was involved in the regulation of mast cell degranulation, we examined only the role of CCK1-R. CCK1-R is mainly involved in gastrointestinal smooth muscle movement and visceral sensory regulation and is closely related to the occurrence and recovery of intestinal paralysis (9).

For the regulatory mechanism between TRPA1 and CCK1-R, in a previous study, researchers found that TRPV1 and TRPA1 were not the key downstream targets in the CCK1-R signalling pathway (38) by detecting whether multiple members of the TRP family were involved in the CCK1-R pathway in neurons. In this study, we incubated mast cells with CCK1-R antagonists and enhancers and then co-cultured them with neurons in a non-contact manner. CCK1-R could not regulate the expression of TRPA1 protein, while TRPA1 could positively regulate the secretion of CCK1-R from dorsal root ganglia, which indicated that the regulation between TRPA1 and CCK1-R was unidirectional.

\section{Limitations}

The limitations of this study are as follows: (I) the early oral nutrition group and TRPA1 inhibitor + early oral nutrition group were designed, but no single TRPA1 inhibitor group was established. Although a significant effect of the TRPA1 inhibitor on mast cell function was observed, the goal of this study was to determine the role of TRPA1 in POI. There are some deficiencies in the mechanism because the effect of early oral nutrition may mask the role of TRPA1 inhibitors. (II) In this study, only histamine was detected as an evaluation of mast cell function and colonic smooth muscle inflammation level. Although histamine is an important mediator that initiates inflammation, evaluating the inflammation response level by using only one indicator is slightly inadequate. However, a previous study focused on inflammation in the smooth muscle layer of the colon and the expression of mast cell-related inflammatory mediators (9). Therefore, no additional mast cell-related inflammatory mediators were designed and detected in this study. (III) Although the results in vitro and in vivo were consistent in this study, cell culture may deviate from the actual situation in vivo.

\section{Conclusions}

In conclusion, early oral nutrition improves POI by the TRPA1/CCK1-R-mediated mast cell-nerve axis. TRPA1 affects mast cell function by regulating CCK1-R, which is one of the mechanisms of TRPA1 in regulating mast cells.

\section{Acknowledgments}

The authors thank Yunnan Labreal Biotechnology Co., Ltd which provided help for detection of some indicators.

Funding: This study was supported by the National Natural Science Foundation of China (NSFC) (No. 81860098), Yunnan Province Joint Special Project of Science \& Technology Department of Yunnan Province and Kunming Medical University (No. 2018FE001-050), Yunnan health training project of high level talents (No. H2017038) and Kunming Medical University training project of 100 talents (No. 60117190466) to DL Sun and Yunnan health training project of high level talents (No. D2017037) to WM Li.

\section{Footnote}

Conflicts of Interest: The authors have no conflicts of interest to declare.

Ethical Statement: The authors are responsible for all aspects of the work in ensuring that questions related to the accuracy or integrity of any part of the work are appropriately investigated and resolved. This study was approved by an institutional ethics committee and followed the tenets of the Declaration of Helsinki. All experiments were conducted in accordance with the care and use of 
laboratory animals published by the Ministry of Science and Technology of China and approved by the Ethics Committee of Kunming Medical University.

Open Access Statement: This is an Open Access article distributed in accordance with the Creative Commons Attribution-NonCommercial-NoDerivs 4.0 International License (CC BY-NC-ND 4.0), which permits the noncommercial replication and distribution of the article with the strict proviso that no changes or edits are made and the original work is properly cited (including links to both the formal publication through the relevant DOI and the license). See: https://creativecommons.org/licenses/by-nc-nd/4.0/.

\section{References}

1. Gero D, Gié O, Hübner M, et al. Postoperative ileus: in search of an international consensus on definition, diagnosis, and treatment. Langenbecks Arch Surg 2017;402:149-58.

2. Peters EG, De Jonge WJ, Smeets BJ, et al. The contribution of mast cells to postoperative ileus in experimental and clinical studies. Neurogastroenterol Motil 2015;27:743-9.

3. Stakenborg N, Wolthuis AM, Gomez-Pinilla PJ, et al. Abdominal vagus nerve stimulation as a new therapeutic approach to prevent postoperative ileus. Neurogastroenterol Motil 2017;29:e13075.

4. Lee YJ, Hussain Z, Huh CW, et al. Inflammation, Impaired Motility, and Permeability in a Guinea Pig Model of Postoperative Ileus. J Neurogastroenterol Motil 2018;24:147-58.

5. de Jonge WJ, The FO, van der Coelen D, et al. Mast cell degranulation during abdominal surgery initiates postoperative ileus in mice. Gastroenterology 2004;127:535-45.

6. The FO, Buist MR, Lei A, et al. The role of mast cell stabilization in treatment of postoperative ileus: a pilot study. Am J Gastroenterol 2009;104:2257-66.

7. Huang Y, Chen SR, Chen H, et al. Endogenous transient receptor potential ankyrin 1 and vanilloid 1 activity potentiates glutamatergic input to spinal lamina I neurons in inflammatory pain. J Neurochem 2019;149:381-98.

8. de Almeida AS, Rigo FK, De Prá SD, et al. Role of transient receptor potential ankyrin 1 (TRPA1) on nociception caused by a murine model of breast carcinoma. Pharmacol Res 2020;152:104576.

9. Lubbers T, Luyer MD, de Haan JJ, et al. Lipid-rich enteral nutrition reduces postoperative ileus in rats via activation of cholecystokinin-receptors. Ann Surg 2009;249:481-7.

10. Ji Y, Sakata Y, Yang Q, et al. Activation of rat intestinal mucosal mast cells by fat absorption. Am J Physiol Gastrointest Liver Physiol 2012;302:G1292-300.

11. de Haan JJ, Hadfoune M, Lubbers T, et al. Lipid-rich enteral nutrition regulates mucosal mast cell activation via the vagal anti-inflammatory reflex. Am J Physiol Gastrointest Liver Physiol 2013;305:G383-91.

12. Tsuchiya K, Ohno N, Kubota K, et al. Activation of TRPA1, but not of TRPV1, improves gut motility in a murine model of postoperative ileus. Gastroenterology 2012;142:S-893.

13. Tsuchiya K, Kubota K, Ohbuchi K, et al. Transient receptor potential ankyrin 1 agonists improve intestinal transit in a murine model of postoperative ileus. Neurogastroenterol Motil 2016;28:1792-805.

14. Nakajima S, Hira T, Yahagi A, et al. Unsaturated aldehydes induce CCK secretion via TRPA1 in STC-1 cells. Mol Nutr Food Res 2014;58:1042-51.

15. Yao PL, Morales JL, Gonzalez FJ, et al. Peroxisome proliferator-activated receptor- $\beta / \delta$ modulates mast cell phenotype. Immunology 2017;150:456-67.

16. Sayed BA, Walker ME, Brown MA. Cutting edge: mast cells regulate disease severity in a relapsingremitting model of multiple sclerosis. J Immunol 2011;186:3294-8.

17. Jyothsna M, Rammanohar M, Kumar K. Histomorphometric Analysis of Angiogenesis using CD31 Immunomarker and Mast Cell Density in Oral Premalignant and Malignant Lesions: A Pilot Study. J Clin Diagn Res 2017;11:ZC37-40.

18. Pongrac JL, Rylett RJ. Molecular mechanisms regulating NGF-mediated enhancement of cholinergic neuronal phenotype: c-fos trans-activation of the choline acetyltransferase gene. J Mol Neurosci 1998;11:79-93.

19. Hsu CC, Lin RL, Lee LY, et al. Hydrogen sulfide induces hypersensitivity of rat capsaicin-sensitive lung vagal neurons: role of TRPA1 receptors. Am J Physiol Regul Integr Comp Physiol 2013;305:R769-79.

20. Shapiro D, Deering-Rice CE, Romero EG, et al. Activation of transient receptor potential ankyrin-1 (TRPA1) in lung cells by wood smoke particulate material. Chem Res Toxicol 2013;26:750-8.

21. Johnson C, Huynh V, Hargrove L, et al. Inhibition of Mast Cell-Derived Histamine Decreases Human Cholangiocarcinoma Growth and Differentiation via c-Kit/Stem Cell Factor-Dependent Signaling. Am J Pathol 2016;186:123-33. 
22. Oka T, Kalesnikoff J, Starkl P, et al. Evidence questioning cromolyn's effectiveness and selectivity as a 'mast cell stabilizer' in mice. Lab Invest 2012;92:1472-82.

23. Moon SJ, An JM, Kim J, et al. Pharmacological characterization of rebamipide: its cholecystokinin CCK1 receptor binding profile and effects on $\mathrm{Ca} 2+$ mobilization and amylase release in rat pancreatic acinar cells. Eur J Pharmacol 2004;505:61-6.

24. Ling X, Westover D, Cao F, et al. Synergistic effect of allyl isothiocyanate (AITC) on cisplatin efficacy in vitro and in vivo. Am J Cancer Res 2015;5:2516-30.

25. Zhang X, Koronowski KB, Li L, et al. Nitro-oleic acid desensitizes TRPA1 and TRPV1 agonist responses in adult rat DRG neurons. Exp Neurol 2014;251:12-21.

26. Mukherjee SP, Lozano N, Kucki M, et al. Detection of Endotoxin Contamination of Graphene Based Materials Using the TNF- $\alpha$ Expression Test and Guidelines for Endotoxin-Free Graphene Oxide Production. PLoS One 2016;11:e0166816.

27. Ceccarelli S, Panera N, Mina M, et al. LPS-induced TNF- $\alpha$ factor mediates pro-inflammatory and profibrogenic pattern in non-alcoholic fatty liver disease. Oncotarget 2015;6:41434-52.

28. Lin CW, Shiosaki K, Miller TR, et al. Characterization of two novel cholecystokinin tetrapeptide (30-33) analogues, A-71623 and A-70874, that exhibit high potency and selectivity for cholecystokinin-A receptors. Mol Pharmacol 1991;39:346-51.

29. Pohl JM, Gutweiler S, Thiebes S, et al. Irf4-dependent CD103+CD11b+ dendritic cells and the intestinal microbiome regulate monocyte and macrophage activation and intestinal peristalsis in postoperative ileus. Gut 2017;66:2110-20.

30. Gottwald TP, Hewlett BR, Lhotak S, et al. Electrical stimulation of the vagus nerve modulates the histamine content of mast cells in the rat jejunal mucosa. Neuroreport 1995;7:313-7.

31. Stead RH, Colley EC, Wang B, et al. Vagal influences over mast cells. Auton Neurosci 2006;125:53-61.

32. Motter AL, Ahern GP. TRPA1 is a polyunsaturated fatty acid sensor in mammals. PLoS One 2012;7:e38439.

33. Yu S, Gao G, Peterson BZ, et al. TRPA1 in mast cell activation-induced long-lasting mechanical hypersensitivity of vagal afferent C-fibers in guinea pig esophagus. Am J Physiol Gastrointest Liver Physiol 2009;297:G34-42.

34. Bell A, Althaus M, Diener M. Communication between mast cells and rat submucosal neurons. Pflugers Arch 2015;467:1809-23.

35. Skaper SD, Facci L, Kee WJ, et al. Potentiation by histamine of synaptically mediated excitotoxicity in cultured hippocampal neurones: a possible role for mast cells. J Neurochem 2001;76:47-55.

36. Kempuraj D, Selvakumar GP, Zaheer S, et al. CrossTalk between Glia, Neurons and Mast Cells in Neuroinflammation Associated with Parkinson's Disease. J Neuroimmune Pharmacol 2018;13:100-12.

37. Juanola C, Giralt M, Jiménez M,et al. Mucosal mast cells are involved in CCK disruption of MMC in the rat intestine. Am J Physiol 1998;275:G63-7.

38. Zhao H, Simasko SM. Role of transient receptor potential channels in cholecystokinin-induced activation of cultured vagal afferent neurons. Endocrinology 2010;151:5237-46.
Cite this article as: Sun DL, Qi YX, Yang T, Lin YY, Li SM, Li YJ, Xu QW, Sun YB, Li WM, Chen XZ, Xu PY. Early oral nutrition improves postoperative ileus through the TRPA1/ CCK1-R-mediated mast cell-nerve axis. Ann Transl Med 2020;8(5):179. doi: 10.21037/atm.2020.01.95 
Supplementary

Table S1 The sequence of primers in this study

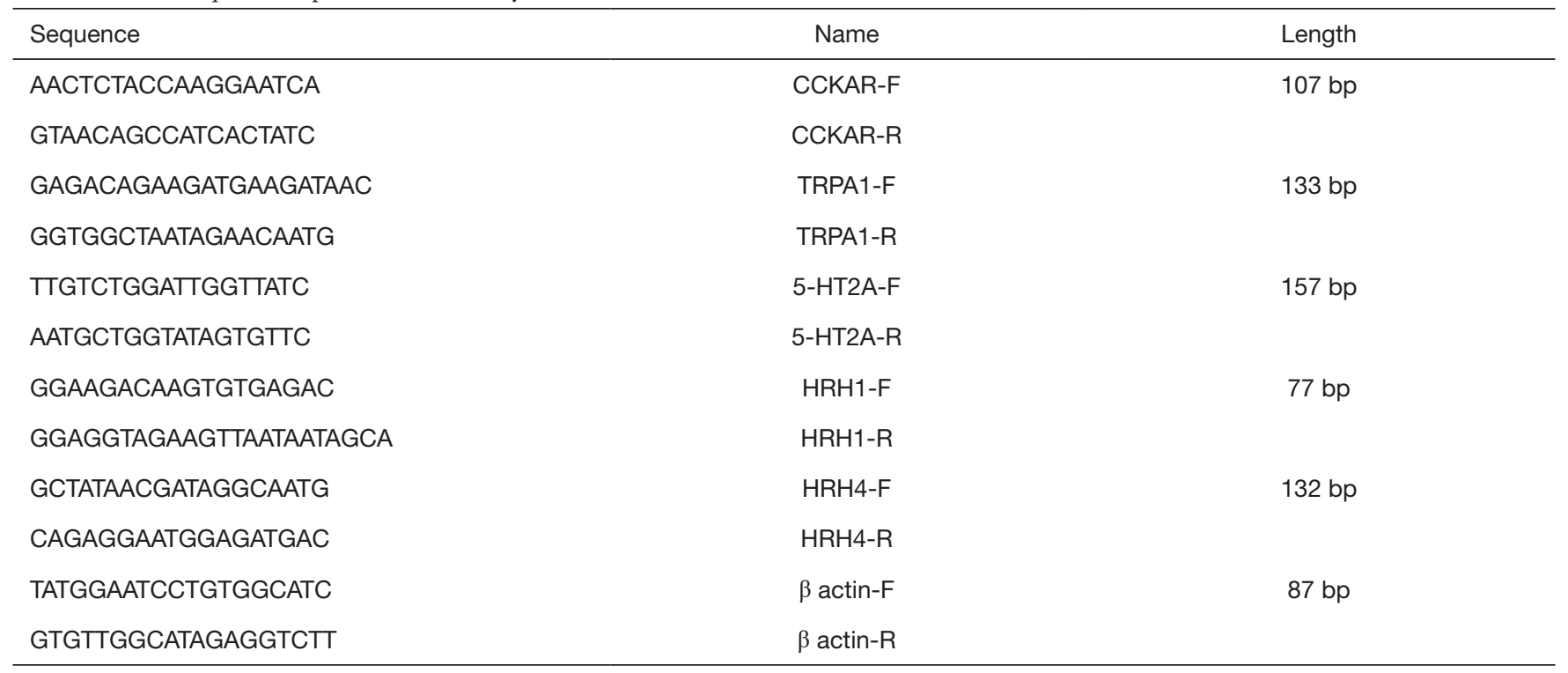

F: forward; R: reverse.
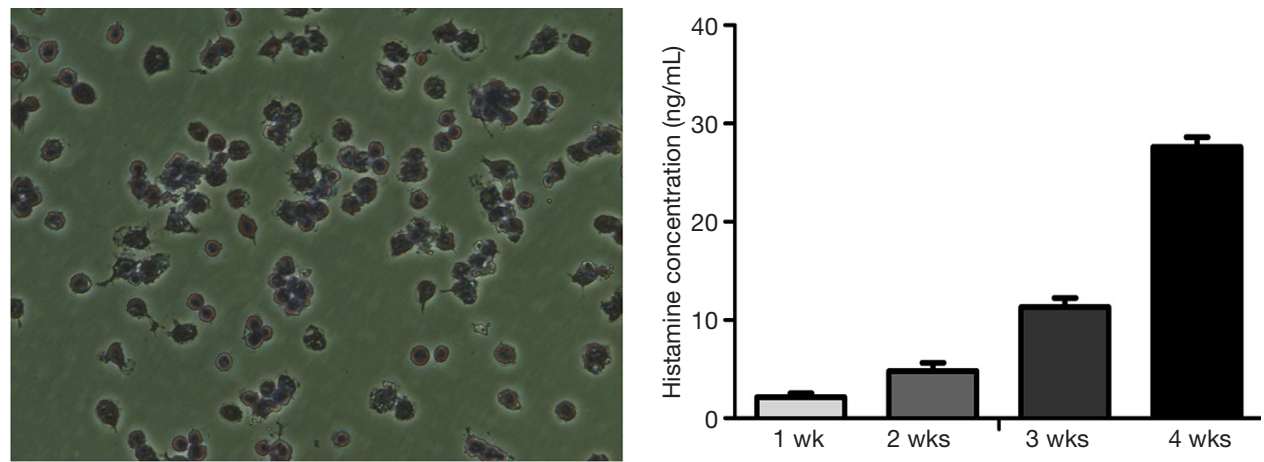

Mature mast cells stained with toluidine blue

Figure S1 Identification of mast cell function $(\times 100)$.
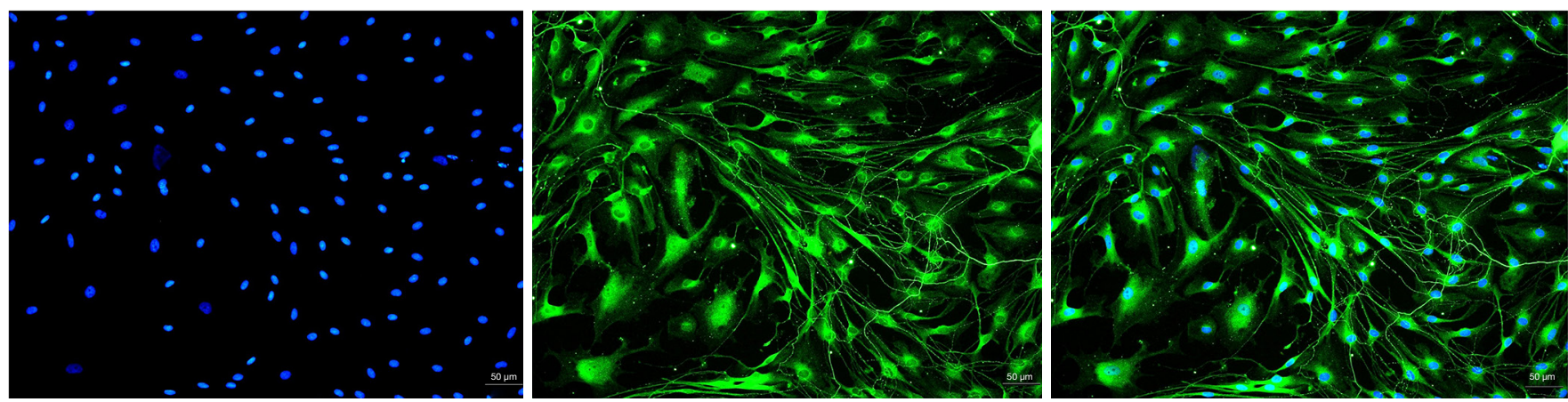

Figure S2 Identification of neurons function. 